\title{
Body in mind
}

\author{
Alexander Jones ${ }^{1 *}$ and Bettina Forster ${ }^{2}$ \\ ${ }^{1}$ Department of Psychology, Middlesex University London, London, UK \\ 2 Department of Psychology, City University London, London, UK \\ *Correspondence: a.j.jones@mdx.ac.uk
}

Edited and reviewed by:

Eddy J. Davelaar, Birkbeck, University of London, UK

Keywords: body in mind, tactile, touch, somatosensory, attention, Somatosensory Evoked Potentials (SEP)

The sense of touch is imperative in cognition, development, and for how we perceive the world and interact with others. The skin envelopes our body and is our largest organ providing us with information about our immediate environment. In addition to this "surface" information, our somatosensory system also processes information regarding body position and internal bodily states. The importance of processing information to our body is unlikely to be disputed, however, relatively little research has focused on the somatosensory system and the body sense as compared to vision and audition. The last two decades have however seen an increased interest in the body sense from a range of different perspectives, using different methodologies which this research topic aimed to reflect.

The broad range of unanswered research questions relating to the body is also mirrored by the contributions in this research topic. We are constantly bombarded with tactile information, such as from the clothes on our body, or the chair we sit upon, yet we are able to process only what is relevant to us. Mechanisms of selective attentions help to prioritize, predict and select information relevant to the situation and to guide our behavior. Selectively attending to a location on the body has repeatedly shown to enhance processing of stimuli at attended over unattended location (e.g., Jones and Forster, 2014). In this research topic, Tamè et al. (2014) showed that detection of a tactile stimulus is dependent upon concurrent presented stimuli which may act as a detection mask, even though the mask is presented at different fingers or hands. Somatosensory information is not typically presented in isolation but the sense of touch is also integrated with other modalities (for an overview see Calvert et al., 2004). Using event-related potentials (ERPs), Staines et al. (2014) showed how visual information relevant to movements modulates somatosensory processing. In a comprehensive review, Heed and Azañón, 2014) explored the findings of how we localize touch to our body. Touch can be localized both using somatotopic but also external coordinates. That is, where on the skin was the stimulus, or where based on external spatial co-ordinates did the tactile stimulus appear. Their overview focuses on how temporal order judgment (TOJ) tasks have been a fruitful paradigm to investigate tactile spatial processing. External and somatotopic maps were also shown, using ERPs, to be affected by where vision is directed (Gherri and Forster, 2014).

Observing others being touched as well as the social aspect of touch are important parts of everyday life and this has also recently attracted increased research interest. In particular, in this research topic Gillmeister (2014) introduced a new behavioral paradigm to measure how observing touch on another person's hand influences the perception of one's own touch. Moreover, Farmer et al. (2014) showed how induced changes in body ownership through observation of touch applied to another person's hand of a different skin color can modulate social attitudes. A similar emerging and interesting research area, briefly covered in this research topic, is how we use interoceptive cues and how the sense of self develops (Sel, 2014). Last but not least, a study investigating the touch and weight perception on aesthetic appreciation points to an important role of one's own body perception in aesthetic perception (Yamada et al., 2014). Together, these recent, seemingly divers studies could fall under the term embodied cognition which now encompasses seemingly diverse fields of psychology highlighting the influence of the body in mind on cognition in general.

This research topic brings together both research from wellestablished paradigms and research areas as well as highlighting how the body and the sense of touch is also being explored in new ways. The contributions here show there are a broad range of perspectives and questions to be addressed when processing stimuli to and within our body. The increased interest in the sense of touch over the last decades will hopefully continue as much still remains to be explored.

\section{REFERENCES}

Calvert, G. A., Spence, C., and Stein, B. E. (2004). The Handbook of Multisensory Processes. Cambridge, MA: The MIT Press.

Farmer, H., Maister, L., and Tsakiris, M. (2014). Change my body, change my mind: the effects of illusory ownership of an outgroup hand on implicit attitudes toward that outgroup. Front. Psychol. 4:1016. doi: 10.3389/fpsyg.2013. 01016

Gherri, E., and Forster, B. (2014). Attention to the body depends on eye-in-orbit position. Front. Psychol. 5:683. doi: 10.3389/fpsyg.2014.00683

Gillmeister, H. (2014). A new perceptual paradigm to investigate the visual remapping of others' tactile sensations onto one's own body shows "mirror touch" for the hands. Front. Psychol. 5:95. doi: 10.3389/fpsyg.2014.00095

Heed, T., and Azañón, E. (2014). Using time to investigate space: a review of tactile temporal order judgments as a window onto spatial processing in touch. Front. Psychol. 5:76. doi: 10.3389/fpsyg.2014.00076

Jones, A., and Forster, B. (2014), Neural correlates of endogenous attention, exogenous attention and inhibition of return in touch. Eur. J. Neurosci. 40, 2389-2398. doi: $10.1111 /$ ejn. 12583

Sel, A. (2014). Predictive codes of interoception, emotion, and the self. Front. Psychol. 5:189. doi: 10.3389/fpsyg.2014.00189

Staines, W. R., Popovich, C., Legon, J. K., and Adams, M. S. (2014). Early modalityspecific somatosensory cortical regions are modulated by attended visual stimuli: interaction of vision, touch, and behavioral intent. Front. Psychol. 5:351. doi: 10.3389/fpsyg.2014.00351 
Tamè, L., Moles, A., and Holmes, N. P. (2014). Within, but not between hands interactions in vibrotactile detection thresholds reflect somatosensory receptive field organization. Front. Psychol. 5:174. doi: 10.3389/fpsyg.2014. 00174

Yamada, Y., Harada, S., Choi, W., Fujino, R., Tokunaga, A., Gao, Y., et al. (2014). Weight lifting can facilitate appreciative comprehension for museum exhibits. Front. Psychol. 5:307. doi: 10.3389/fpsyg.2014. 00307

Conflict of Interest Statement: The authors declare that the research was conducted in the absence of any commercial or financial relationships that could be construed as a potential conflict of interest.
Received: 17 December 2014; accepted: 12 January 2015; published online: 30 January 2015.

Citation: Jones A and Forster B (2015) Body in mind. Front. Psychol. 6:56. doi: 10.3389/fpsyg.2015.00056

This article was submitted to Cognitive Science, a section of the journal Frontiers in Psychology.

Copyright (C) 2015 Jones and Forster. This is an open-access article distributed under the terms of the Creative Commons Attribution License (CC BY). The use, distribution or reproduction in other forums is permitted, provided the original author(s) or licensor are credited and that the original publication in this journal is cited, in accordance with accepted academic practice. No use, distribution or reproduction is permitted which does not comply with these terms. 